\title{
Response to comment on "Point of zero charge of a corundum-water interface probed with optical second harmonic generation (SHG) and atomic force microscopy (AFM): New approaches to oxide surface charge"
}

\author{
Andrew G. Stack, Steven R. Higgins, and Carrick M. Eggleston* \\ Department of Geology and Geophysics, University of Wyoming, Laramie, WY 82071-3006, USA
}

(Received February 11, 2002; accepted in revised form May 30, 2002)

\section{INTRODUCTION}

The purpose of this response is to address several misrepresentations of Stack et al. (2001) and its intent in the preceding Comment. At issue, primarily, is our observed point of zero salt effect (p.z.s.e.) of a single crystal face of corundum using optical second harmonic generation $(\mathrm{SHG})$ at a $\mathrm{pH}(\sim 5)$ much lower than the accepted value (in the $8-9$ range) of the pristine p.z.c. of alumina powders. Dr. Kosmulski's main claim is that our anomalous observation is the result of surface contamination.

Dr. Kosmulski asserts that "Stack et al. (2001) challenged the well-established PZC and suggested the pristine point of zero charge of aluminum oxide at $\mathrm{pH} 5$ to 6 . For an unaware reader, the message of the abstract and conclusions of their paper may be that the well-established value $(\mathrm{pH} 9)$ should be abandoned and the new value ( $\mathrm{pH} 5$ to 6) should be used instead." This is an indiscriminate misrepresentation of Stack et al. (2001); we never claimed that our value should be taken as "definitive"; our paper clearly recognizes the difference between our observation and the "accepted" pzc, and our paper devotes a substantial amount of text to exploring possible reasons for the difference. Stack et al. (2001) does not state in any manner that anyone should abandon the well-established value. Dr. Kosmulski's "unaware reader" may, however, conclude from the misrepresentations in the Comment that we made such statements in Stack et al. (2001).

The purpose of Stack et al. (2001) was not to assert a new, definitive pristine p.z.c. for alumina, but rather to show how the SHG technique could be used to obtain information about electric fields at the surface of insulating minerals without the need to interpret titration stoichiometry. Dr. Kosmulski's point is mainly that (a) silicate, sulfate, or some other contaminant could adsorb to our surface and produce a significant shift in the $\mathrm{pH}$ of the p.z.c., or (b) the alumina itself is impure and could exhibit a p.z.c. substantially different from that of a pure alumina. We agree - and our manuscript does not say otherwise. Our abstract plainly states that the SHG (and AFM) results are different from expectations and points out that the reasons for the differences are unclear. In the last paragraph of section 4.1 of Stack et al. (2001), we specifically discuss the possible role of contaminants in shifting the p.z.c. to lower $\mathrm{pH}$ values. The surface studied was not necessarily "pristine," and our paper recognizes this fact repeatedly. We refer to our observations as pertaining to the p.z.c. and never as an indication of the

* Author to whom correspondence should be addressed (carrick@ uwyo.edu). p.z.n.p.c. We have made a distinction between the sample we studied (which is nominally a basal surface of alumina but with a $5^{\circ}$ miscut and numerous polishing scratches) and the "clean alumina powder[s]" of the consensus (Stack et al., 2001).

Dr. Kosmulski does a particular disservice with the statement “... the discrepancy between the results of Stack et al. (2001) and those published by others indicates certain limitations of the Second Harmonic Generation (SHG) method as the tool to establish the pristine PZC of alumina (and other metal oxides)." In fact, Dr. Kosmulski's primary scientific objections have nothing to do with the SHG technique per se but rather with the wet-chemical system to which SHG was applied. This is an important distinction. The SHG is showing us that a point of zero salt effect does indeed occur in the region of $\mathrm{pH} 5$ for the surface in question. It is a self-contradiction to impugn the SHG technique and then base one's objections to the chemistry on the correctness of the SHG results.

The discrepancy between our SHG results and the accepted value for the pristine p.z.c. was indeed a source of concern during our experimentation, and it prompted us to examine the surface composition using Auger Electron Spectroscopy (AES) after the SHG experiments in an attempt to identify contaminants that could have caused such a shift. As stated in section 3.3 of our paper, we did not detect anything other than $\mathrm{Al}, \mathrm{O}$, and adventitious $\mathrm{C}$ (which is ubiquitous in surface analyses; see, e.g., Hochella, 1988). Any contaminants other than adventitious carbon were therefore undetectable by AES. Could Si have been present in sufficient quantity to lower the p.z.c. to $\mathrm{pH}$ 5 but remain undetectable? The most prominent Si LMM peak (i.e., that with the greatest sensitivity factor for $\mathrm{Si}$ ) at $92 \mathrm{eV}$ would have an escape depth of $\sim 1.0$ to $1.5 \mathrm{~nm}$ (Hochella, 1988; Bishop, 1989). For simplicity, let us assume $1.3 \mathrm{~nm}$ (one unit cell). Let us choose for comparison kaolinite, a material composed of silica and alumina with a p.z.c. near that which we observed ( $\mathrm{pH}$ 5.2; Schroth and Sposito, 1997). If our surface was kaolinite-like, with a half-monolayer of silica, we would have roughly 3.33 atom percent $\mathrm{Si}$ within the analyzed surface volume. This amount of Si should be evident in the AES, given an accepted sensitivity of 0.1 to 1 atom percent (Bishop, 1989). We detected no hint of an $\mathrm{Si}$ peak. If we assume a greater escape depth (i.e., doubling it to $2.6 \mathrm{~nm}$ ), the atom percent decreases to $1.67 \%$, but this amount should still be detectable.

A reviewer has argued that the positive surface charge at $\mathrm{pH}$ 5 on alumina (with a p.z.c. at 8 or 9) could be compensated by a much smaller surface concentration of anions than we have assumed above if each compensating ion held one negative charge. This argument is not compelling for silica, which has 
very low negative surface charge density at $\mathrm{pH} 5$, but could possibly apply to sulfate/bisulfate.

Thus, in Stack et al. (2001) we could not conclude that contaminants were present in sufficient quantity to cause the difference between our observed p.z.c. and that accepted for clean powders. However, in our view, the amounts needed to shift the p.z.c. are also close enough to detection limits to preclude an unequivocal conclusion. Our main point is that we did indeed consider a number of possible contaminants, and our paper states as much. We would have liked to have found an obvious explanation for the discrepancy between our p.z.c. and the accepted one, but such an explanation is elusive. We simply could not prove or disprove that surface contamination was necessarily the cause. The wording in our paper is specifically intended to avoid committing to a particular explanation, because there remained several possibilities.

Dr. Kosmulski dismisses the fact that we are not dealing with a powder but with a single crystal surface while referring to the MUSIC model of Hiemstra et al. (1989a, 1989b). We cannot exclude the possibility that the corundum basal plane behaves more acidically than other crystal faces. For example, if $\mathrm{Al}$ in trigonal basal plane surface sites is predominantly tetrahedral in the presence of aqueous solution, this might have a dramatic effect on p.z.c. relative to aluminas with predominantly octahedral aluminum (e.g., Parks, 1967).

Others have observed a p.z.c. at around pH 5 specifically for a corundum basal plane (Larson et al., 1997; electrophoresis: Smit and Holden, 1980; Smit and Stein, 1976). Larson et al. (1997) find a value similar to ours specifically for a basal plane and document precautions against silica contamination. Dr. Kosmulski, deprived of the "silica problem" in this case, refers to possible (but undocumented) surfactant adsorption instead. This was our point all along - having looked for specific contaminants spectroscopically, we could not find them, so we avoided attributing our observed p.z.c. to a specific cause. Dr. Kosmulski has in effect taken the same approach.

Dr. Kosmulski states, in reference to the model used to calculate surface potentials from SHG data, that "This resulted in strong dependence of the calculated surface potential on the nature of the co-ions. ..." Surface potential should indeed be independent of the identity of non-specifically adsorbing ions, but there are other reasons for these differences that have to do with SHG as opposed to a titration. The calculated surface potentials are based on the Gouy-Chapman model that is internally calibrated at a $\mathrm{pH}$ for which surface potential changes only with ionic strength (the density of charged surface sites is constant). As discussed in Stack et al. (2001), this requirement may not have been entirely satisfied, which would cause overestimation of surface potentials. Moreover, the SHG theory described in Ong et al. (1992) and Zhao et al. (1993) predicts a linear relationship between SH field strength and surface potential. However, Gragson et al. (1997) show that a linear relationship is not necessarily adequate at high surface potentials. Because the model is calibrated at high surface potentials, other possible errors are introduced and were discussed in Stack et al. (2001). Variation in calculated surface potential with varying electrolyte is not necessarily real but could stem from the calibration of the model between experiments and our assumptions regarding how water molecules respond to a strong electric field. To be clear, however, at the p.z.c., SH intensity will still be invariant with ionic strength, because there is no net surface electric field and, therefore, no need to assume a specific relationship between the $\mathrm{SH}$ electric field and the surface potential. The crossover points in Stack et al. (2001) are not the result of the application of a model, but are a simple experimental observation.

In conclusion, we are exploring new techniques to better understand mineral surface chemistry at a fundamental level. SHG is one of the few ways in which we can probe the oxide-water interface in an intrinsically surface-sensitive manner. SHG was used in Stack et al. (2001) and in other studies to probe surface electric fields, and there are many other interesting questions regarding the adsorption of organic molecules to mineral surfaces that can be addressed with this technique. In Stack et al. (2001), we were unable to prove that we had a contaminant or that the intrinsic chemistry of the basal corundum surface caused the discrepancy between our observed p.z.c. and the accepted value. Dr. Kosmulski's Comment brings up some possibilities, but after investigating his assertions, we are left where we started-unable to prove either possibility. None of this, however, should detract from the SHG technique as a useful probe for the mineral-water interface.

Acknowledgments - We thank Dimitri A. Sverjensky for his fair and careful handling of this Comment/Response.

\section{Associate editor: D. A. Sverjensky}

\section{REFERENCES}

Bishop H. E. (1989) Auger electron spectroscopy. In Methods of Surface Analysis, Techniques and Applications (ed. J. M. Walls), pp. 87-126. Cambridge University Press, Cambridge.

Gragson D. E., McCarty B. M., and Richmond G. L. (1997) Ordering of interfacial water molecules at the charged air/water interface observed by vibrational sum frequency generation. J. Am. Chem. Soc. 119, 6144-6152.

Hiemstra T., de Wit J. C., and van Riemsdijk W. H. (1989a) Multisite proton adsorption modeling at the solid solution interface of (hydr)oxides: A new approach II. Applications to various important (hydr)oxides. J. Colloid Interf. Sci. 133, 105-117.

Hiemstra T., van Riemsdijk W. H., and Bolt G. H. (1989b) Multisite proton adsorption modeling at the solid solution interface of (hydr)oxides: A new approach I. Model description and evaluation of reaction constants. J. Colloid Interf. Sci. 133, 91-104.

Hochella M. F. Jr. (1988) Auger electron and X-ray photoelectron spectroscopies. In Spectroscopic Methods in Mineralogy and Geology (ed. F. C. Hawthorne). Rev. Miner. 18, 573-637.

Larson I., Drummond C. J., Chan D. Y. C., and Grieser F. (1997) Direct force measurements between silica and alumina. Langmuir 13, $2109-2112$.

Ong S., Zhao X., and Eisenthal K. B. (1992) Polarization of water molecules at a charged interface: Second harmonic studies of the silica/water interface. Chem. Phys. Lett. 191, 327-335.

Parks G. A. (1967) Aqueous surface chemistry of oxides and complex oxide minerals. In Equilibrium Concepts in Natural Water Systems (ed. W. Stumm), Adv. Chem. Ser. 67, 121-160.

Schroth B. and Sposito G. (1997) Surface charge properties of kaolinite. Clay. Clay Miner. 45, 85-91.

Smit W. and Stein C. L. M. (1976) Zeta-potential and radiotracer adsorption measurements on EFG $\alpha-\mathrm{Al}_{2} \mathrm{O}_{3}$ single crystals in $\mathrm{NaBr}$ solutions. J. Colloid Interf. Sci. 78, 1-14.

Smit W. and Holden H. N. (1980) Electroosmotic zeta potential measurements on single crystals. J. Colloid Interf. Sci. 60, 299-307.

Stack A. G., Higgins S. R., and Eggleston C. M. (2001) Point of zero charge of a corundum-water interrface probed with optical Second Harmonic Generation (SHG) and Atomic Force Microscopy (AFM): New approaches to oxide surface charge. Geochim. Cosmochim. Acta 65, 3055-3063.

Zhao X., Ong S., and Eisenthal K. B. (1993) Polarization of water molecules at a charged interface. Second harmonic studies of charged monolayers at the air/water interface. Chem. Phys. Lett. 202, 513-520. 